\title{
Wavelength drift of PMMA-based optical fiber Bragg grating induced by optical absorption
}

\author{
Wei Zhang, Ada Abang, David J. Webb, and Gang-Ding Peng
}

\begin{abstract}
The transmission loss in polymer optical fiber is much higher than that in silica fiber. Very strong absorption bands dominate throughout the visible and near infrared. Optical absorption increases the internal temperature of the polymer fiber and reduces the wavelength of any polymer optical fiber Bragg grating inscribed within the fiber. In this work we have investigated the wavelength drift of FBGs inscribed in poly(methyl methacrylate) (PMMA) based fiber under illumination at different wavelengths. The experiments have shown that the characteristic wavelength of such a POFBG starts decreasing after a light source is applied to it. This decrease continues until equilibrium inside the fiber is established, depending on the surrounding humidity, the optical power applied, and operation wavelength.
\end{abstract}

Index Terms - Fiber Bragg gratings, Photothermal effects Polymer optical fiber, Humidity dependence.

\section{INTRODUCTION}

$\mathrm{P}$ OLYMER OPTICAL fibers (POFs) provide a lower cost alternative to silica optical fibers. As sensors, POFs have additional advantages, including high strain limits, high fracture toughness, high flexibility in bending, large negative thermo-optic coefficients and for some materials an affinity for water. Moreover polymers have excellent compatibility with organic materials, giving them great potential for biomedical applications [1].

Compared to its silica counterpart polymer optical fiber shows high transmission loss. The primary sources of absorption in polymer optical fibers are vibrations and electronic transitions of molecular groups. This is an inherent property and can vary with the type of materials. Many polymers are capable of absorbing a certain amount of water and this is true of one of the most common materials from which POF is fabricated: poly (methyl methacrylate) (PMMA). The affinity for water in PMMA leads to a swelling of the fiber and an increase of refractive index [2], both of which contribute to an increase in the Bragg wavelength of a FBG written in the PMMA based optical fiber [3]. This is a potentially very useful property, and has been used to construct an optical fiber humidity sensor [4] which has

Wei Zhang, Ada Abang, David J. Webb are with Aston Institute of Photonic Technology, Aston University, Birmingham, B4 7ET, UK (e-mail: w.zhang@aston.ac.uk).

Gangding Peng is with the School of Electrical Engineering and Telecommunications, University of New South Wales, Sydney, NSW 2052, Australia. possible applications in the chemical processing, agriculture, food storage, paper manufacturing, semiconductor and pharmaceutical industries, where humidity is monitored and controlled to ensure product quality. PMMA based optical fiber Bragg grating (POFBG) has recently been applied, for example, to quantifying the small amount of water present in aviation fuel [5]. However, water content inside PMMA optical fiber introduces a large transmission loss, too, due to the $\mathrm{O}-\mathrm{H}$ stretching vibration. It has been reported that absorbed water becomes a significant constituent for the loss of POFs, and the amount of loss due to water absorption depends on the relative humidity of the environment surrounding POFs [6]. In this paper we examine the consequences of the high attenuation of POF as it relates to grating interrogation. We find that illumination of the POFBG can cause a significant wavelength drift due to the photothermal effect in the fiber. Furthermore, for PMMA based fiber, the wavelength drift depends on the humidity environment of the fiber. We identify operating regimes which minimize these effects.

\section{PRINCIPLE}

The Bragg wavelength of a PMMA based fiber grating depends on the effective core refractive index, $n_{\text {eff }}$, and the grating pitch, $\Lambda$, both of which are a function of temperature $T$ and the water content $w$. For a PMMA based fiber grating the Bragg wavelength can then be expressed as

$$
\lambda_{B}=2 n_{\text {eff }}(T, w) \Lambda(T, w)
$$

For constant temperature, the Bragg wavelength change of a POFBG against humidity can be expressed as

$$
\Delta \lambda_{B}=\lambda_{B}(\eta+\beta) \Delta H
$$

where $\lambda_{B}$ is the initial Bragg wavelength, $\eta$ is the normalized refractive index change with humidity $(\% \mathrm{RH})^{-1}$ and $\beta$ the swelling coefficient related to humidity induced volumetric change $(\% \mathrm{RH})^{-1}$. For constant relative humidity $(\mathrm{RH})$ the Bragg wavelength change as a result of varying temperature, can be expressed as

$$
\Delta \lambda_{B}=\lambda_{B}(\alpha+\xi) \Delta T
$$

where $\alpha$ is the thermal expansion coefficient, $\xi$ the normalized thermo-optic coefficient.

In general the refractive index and the grating period can be modulated by external changes such as temperature, humidity, and strain. However some internal changes may also vary the Bragg wavelength of a POFBG. The core of polymer fiber is 
made of organic materials (polymers) and vibrations of molecular groups such as carbon-hydrogen $(\mathrm{C}-\mathrm{H})$ and oxygen-hydrogen $(\mathrm{O}-\mathrm{H})$ are the fundamental origin of the absorption loss [7]. A large part of the absorption of optical energy in those spectral bands will convert into thermal energy, which is described as photothermal effect. It is well known that the thermo-optic coefficient of PMMA is negative [8] so the generated heat, which raises the internal temperature in POFBG, introduces a negative change of refractive index of the fiber core as well as causing thermal expansion of the grating period [4]. Since thermo-optic effect is dominant in many polymer optical fibers, the Bragg wavelength of the POFBG decreases with the generated heat produced by the absorbed optical energy. This change may be easily missed when the grating is being characterized in the laboratory and lead to uncertainty in quantifying POFBG sensor performance.

\section{EXPERIMENTAL RESULTS}

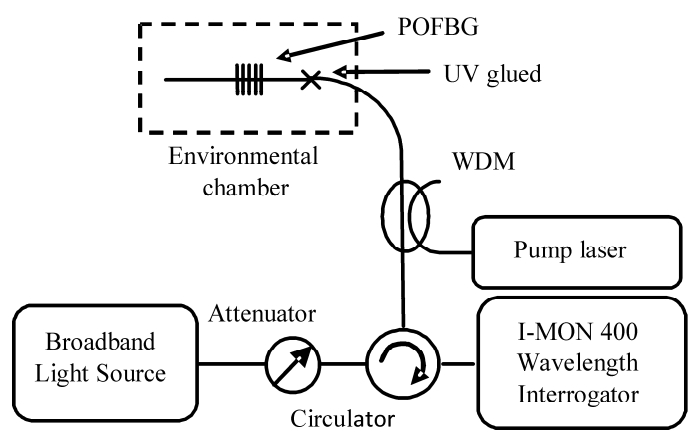

Fig. 1. Experimental arrangement for investigating POFBG wavelength drift induced by optical absorption

An experiment was set up to investigate this mechanism, as shown in Fig. 1. Due to the high optical loss of POF in the $1550 \mathrm{~nm}$ region [9] a short length of POF is generally used to construct a POFBG sensor, which is then connected to a standard single-mode silica fiber down-lead. POF cannot be cleaved in the same fashion as silica fiber, neither can it be sliced using a fusion splicer. A UV gluing technique was developed [10] in which POF is cut by using hot knife to get a clean end face, then butt-coupled with a single-mode silica fiber angled pigtail; the butt-coupling joint is glued with a UV curable glue (Norland 76). The PMMA based POF contained a $10 \mathrm{~mm}$ long FBG, fabricated by illuminating from above a phase mask placed on top of the POF using 325nm UV light from a HeCd laser. The PMMA based optical fiber was core doped to improve its photosensitivity [10]. The fiber has a core of $\sim 9 \mu \mathrm{m}$ in diameter and a cladding of approximately $190 \mu \mathrm{m}$ [11]. The POFBGs were placed inside an environmental chamber (Sanyo Gallenkamp) to allow operation in a controlled temperature and humidity environment. The wavelengths of the POFBGs were monitored by using an IBSEN I-MON 400 wavelength interrogation system. A fiber attenuator was inserted in between the broadband source (Thorlab ASE730) and the circulator to adjust the input optical power to the POFBG. The
POFBG was connected to port 2 of the circulator via a WDM fiber coupler (which was later used to connect a pump laser at a different wavelength in order to investigate the effect in different wavelength regions). The input optical power can be measured at the exit of the WDM coupler before it is launched into the POFBG. It, however, should be pointed out that this measured power is only the power exiting from the WDM coupler rather than the received power by the polymer optical fiber, since the UV glued joint introduces a significant insertion loss.

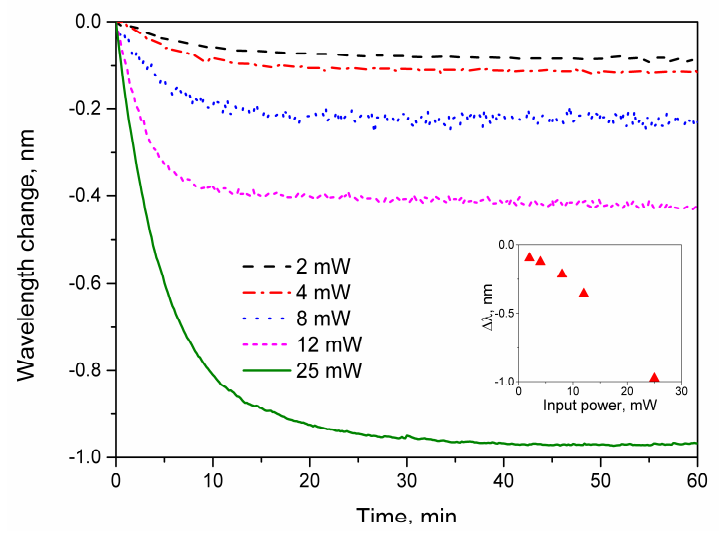

Fig. 2. Time responses of POFBG wavelength at $45 \% \mathrm{RH}, 23^{\circ} \mathrm{C}$. Inset: POFBG wavelength drift against input powers at $45 \% \mathrm{RH}$.

A POFBG was placed in the environmental chamber set at constant temperature and humidity $\left(23^{\circ} \mathrm{C}\right.$ and $\left.45 \% \mathrm{RH}\right)$. The wavelength interrogation system started to record the POFBG wavelength from the switch-on of the broadband light source. POFBGs were first tested under different input optical powers to investigate the time response of the grating wavelength. Typical time responses of the wavelength drifts of one POFBG were captured and are plotted in Fig. 2. The wavelength of the POFBG decreases with time from switch-on till equilibrium is established. The final wavelength reduction increases with the input optical power level. The wavelength drifts against input power are plotted in the inset of Fig. 2.

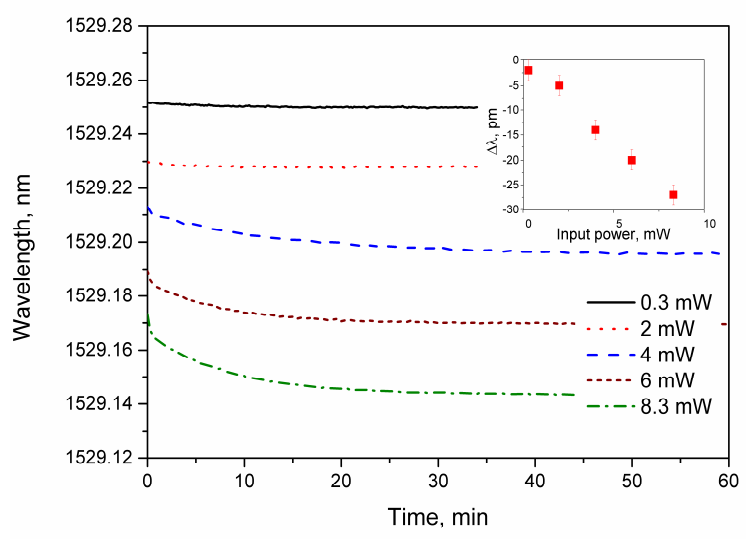

Fig. 3. Typical time responses of POFBG wavelength at $1.3 \% \mathrm{RH}, 23^{\circ} \mathrm{C}$ (traces are vertically offset to give a clear display). Inset: POFBG wavelength drift against input power at $1.3 \% \mathrm{RH}$.

The refractive index and volume of PMMA based optical fiber varies with surrounding relative humidity. This consequently leads to a wavelength change of a POFBG, as 
defined by (2). However, water content inside PMMA optical fiber introduces additional transmission loss due to the $\mathrm{O}-\mathrm{H}$ stretching vibration. As reported in [6] the absorption peak of the $5^{\text {th }}$ harmonic of $\mathrm{O}-\mathrm{H}$ stretching vibration $\left(5 \mathrm{v}_{\mathrm{OH}}\right)$ at $614 \mathrm{~nm}$ was not detectable in a dry environment; however, a loss increment as high as $30 \mathrm{~dB} / \mathrm{km}$ was measured when the humidity was raised to $90 \% \mathrm{RH}$. The loss increment at $746 \mathrm{~nm}$ $\left(4 \mathrm{v}_{\mathrm{OH}}\right)$ was measured to be as high as $600 \mathrm{~dB} / \mathrm{km}$. The water induced loss increment in the $1550 \mathrm{~nm}$ region has not been reported. However, one expects significant loss increment around $1550 \mathrm{~nm}$ as the tail of absorption band of $2 \mathrm{v}_{\mathrm{OH}}$ extends to this region. This implies a significant change in intrinsic optical absorption induced by water content in PMMA based optical fiber. The same POFBG as used in the previous experiment was placed in the environmental chamber with the temperature kept constant (at $23^{\circ} \mathrm{C}$ ) and the humidity set to different values. In the experiments the minimum humidity the environmental chamber could reach at that temperature was $20 \%$, so a low humidity environment was created by using a sealed chamber containing desiccant. A POFBG and the probe of a thermo hygrometer (Testo 625) were inserted into the desiccator through air-tight seals. The desiccant provided an environment of $23{ }^{\circ} \mathrm{C}$ and $1.3 \%$ relative humidity. Different optical power was applied to the POFBG by adjusting the attenuator. The recorded wavelength time responses and the relative wavelength drifts at different input power levels at such a low humidity are shown in Fig. 3.

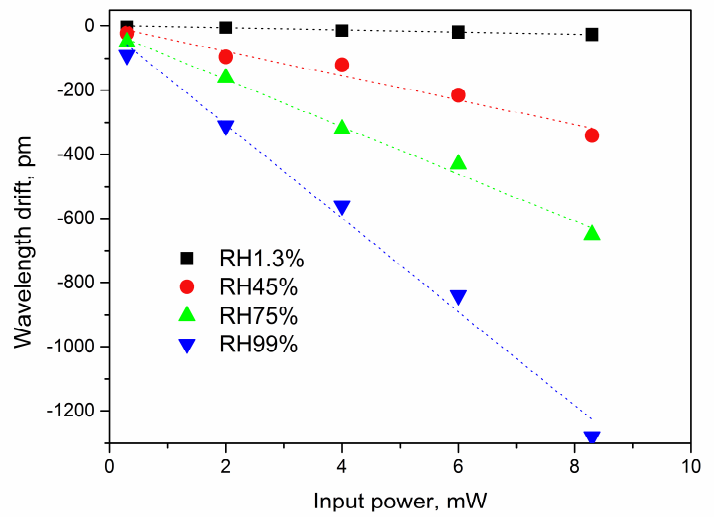

(a)

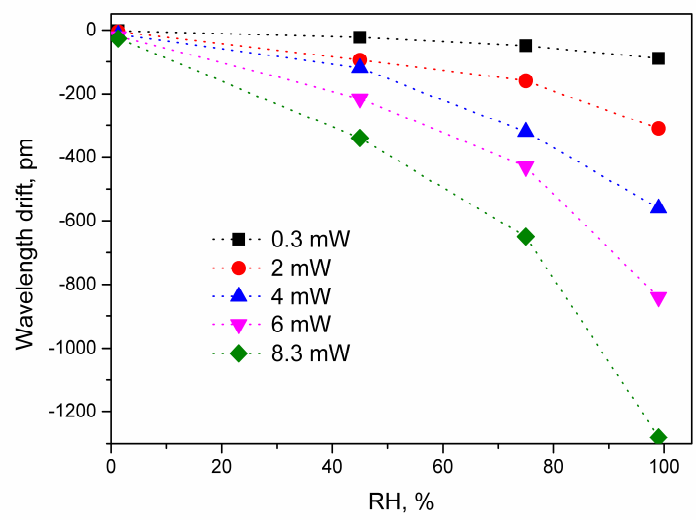

(b)

Fig. 4. POFBG wavelength drift (a) against input power, (b) against relative humidity level.
The responses of the POFBG under different input power at different relative humidity levels were also measured. The corresponding wavelength drifts against input power and relative humidity are summarized in Fig. 4 . It shows that the wavelength reduction of the POFBG at a constant humidity level increases almost linearly with the increment of input power. This corresponds to internal temperature change inside the POF. It performs like a POFBG temperature sensor in which POFBG wavelength varies linearly with temperature in a certain range [12]. However, the wavelength reduction of the POFBG under a certain input power varies nonlinearly with the relative humidity. This behavior is in agreement with the nonlinear loss increment of POF with the increase of relative humidity [6]. In [6] it was found that the loss of POF increased near-logarithmically with the increase of relative humidity. It should be stressed that the wavelength reduction in Fig. $4 \mathrm{~b}$ is only related to the optical absorption in POF at different humidity levels and is not a POFBG humidity sensing response. Indeed the POFBG wavelength actually increases with relative humidity [4, 12], as defined by (2).

At a low humidity level the wavelength drift of the POFBG induced by the photothermal effect is greatly reduced. At a low input power $(0.3 \mathrm{~mW})$ and low humidity level $(1.3 \% \mathrm{RH})$ the wavelength drift of the POFBG is about $-2 \mathrm{pm}, 1$ hour after light source switch-on. It indicates that water content inside PMMA optical fibers plays an important role in this photothermal effect as it changes the intrinsic optical absorptions. The wavelength drift induced by the photothermal effect is minimized at low relative humidity and low input optical power.

Different WDM couplers and pump lasers were then used, as shown in Fig.1, to look into the photothermal effects of POFBGs operating in different wavelength bands. We first turned on the broadband source and monitored the time response of the POFBG wavelength until it reached a stable value during which the POFBG was kept in the environmental chamber $\left(23^{\circ} \mathrm{C}\right.$ and $\left.45 \% \mathrm{RH}\right)$. Depending on the spectral region of interest, one of 4 laser diodes was then used in turn. By setting the output optical power of the laser diode to a fixed level $(5 \mathrm{~mW})$ we could obtain the POFBG wavelength drift influenced by pump lasers at different wavelengths. The sensitivity of the POFBG wavelength to pumping power at the different wavelengths is summarized in Table I.

TABLE I

POFBG WAVELENGTH PUMPING SENSITIVITY WHEN PUMPED AT DIFFERENT WAVELENGTHS

\begin{tabular}{cc}
\hline \hline & \\
Laser wavelength $(\mathrm{nm})$ & Wavelength drift $(\mathrm{pm} / \mathrm{mW})$ \\
808 & $-5 \pm 3$ \\
980 & $-5 \pm 3$ \\
1330 & $-12 \pm 5$ \\
1480 & $-60 \pm 10$ \\
\hline \hline
\end{tabular}

From the summarized results in Table I for the same pump power, it may be seen that the introduced wavelength drift at $808 \mathrm{~nm}$ is much lower than that at $1480 \mathrm{~nm}$. This is in agreement with the lower transmission loss of POF at the shorter wavelengths [7]. 


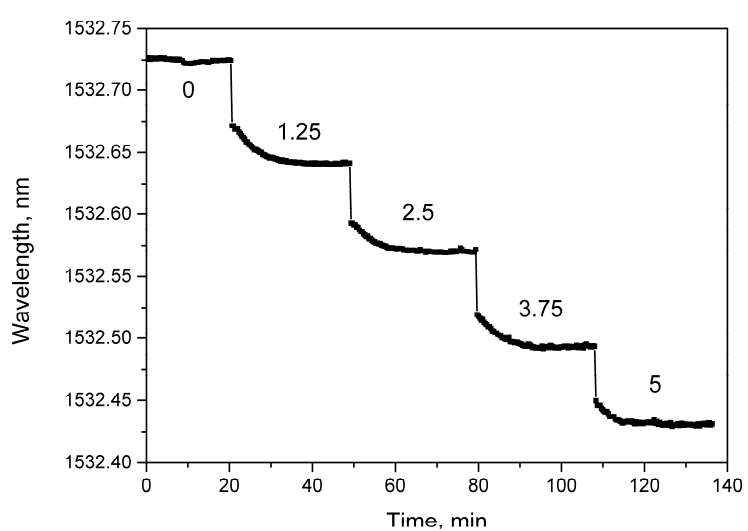

Fig. 5 POFBG wavelength response pumped by a $1480 \mathrm{~nm}$ laser diode

The pump source power sensitivity studied here can be an advantage in some applications when the wavelength of the grating in use needs to be remotely tuned. For example, the wavelength of a POFBG written in the $\mathrm{C}$ band could be remotely adjusted by a pump laser in the $\mathrm{L}$ band or vice versa. This provides a potential approach to create a reconfigurable optical network. A recorded time response of the POFBG is shown in Fig. 5 while the output power of the laser diode with a wavelength of $1480 \mathrm{~nm}$ was step-changed. When the pumping power varied from 0 to $5 \mathrm{~mW}$ the overall wavelength reduction was $294 \mathrm{pm}$, indicating a ratio of $-59 \mathrm{pm} / \mathrm{mW}$ at this wavelength.

\section{CONCLUSION}

It should be pointed out that since additional dopants or copolymers are used to control the core index of step index fibers, the performance of PMMA based fiber gratings may vary with the type of fiber used. The results obtained indicate that the time to reach equilibrium is proportional to the input optical power and the surrounding humidity. The equilibrium time can also be expected to depend on the diameter of POF similar to the response time for POFBG humidity sensors [12]. The insertion loss induced by UV gluing and grating position can be different for different POFBGs, resulting in different optical powers arriving at the grating when used in the set up depicted in Fig 1. Consequently, the results presented here were obtained from the same POFBG though the results from other POFBGs showed similar tendencies [13].

The input optical power to a POFBG plays an important role in the characteristic wavelength of the POFBG due to the photothermal effect of absorbed optical power. The wavelength drift introduced by this optical absorption depends on the wavelength of the illuminating light, the optical power and - for PMMA based fiber - the surrounding humidity. Because of this last dependence, the issue cannot be circumvented by simply waiting for equilibrium to be reached, since a change in the external humidity will lead to an error in the recovered wavelength. Consequently, in many applications it will be necessary to use an interrogating wavelength below 1 micron. The photothermal effect will be minimized if the POFBG is subject to low illumination power and operated in a dry environment. Additional benefits would arise through the use of fiber based on TOPAS, which displays negligible humidity sensitivity [14]. Whilst the effects discussed in this paper are mostly noticeable in PMMA based optical fiber gratings. It is also applicable to silica fiber gratings where the much lower absorption loss renders the problem inconsequential except when very high powers are used [15].

\section{REFERENCES}

[1] D. J. Webb, and K. Kalli, "Polymer Fiber Bragg Gratings" in Fiber Bragg Grating Sensors, A. Cusano, A. Cutolo and J. Albert ed., Bentham Science Publishers Ltd, 2011.

[2] T. Watanabe, N. Ooba, Y. Hida, and M. Hikita, "Influence of humidity on refractive index of polymers for optical waveguide and its temperature dependence", Appl. Phys. Lett., vol. 72, p. 1533, 1998.

[3] N. G. Harbach, "Fibre bragg gratings in polymer optical fibres." Ph.D. dissertation, EPFL, Lausanne, 2008.

[4] C. Zhang, W. Zhang, D. J. Webb, and G.-D. Peng, "Optical fibre temperature and humidity sensor," Electron. Lett., vol. 46 pp. 643-644 Apr. 2010.

[5] C. Zhang, X. Chen, D. J. Webb, and G.-D. Peng, "Water detection in jet fuel using a polymer optical fibre Bragg grating", Proc. SPIE, vol. 7503, p. $750380-1$, Oct. 2009.

[6] T. Kaino, "Influence of water absorption on plastic optical fibers", Appl. Opt., vol. 24, pp. 4192-4195, 1985

[7] T. Kaino, "Polymer Optical Fibers" in Polymers for Lightwave and Integrated Optics, L. A. Hornak ed., New York: Marcel Dekker, Inc., 1992.

[8] M. B. J. Diemeer, "Polymeric thermo-optic space switches for optical communications", Optical Materials vol. 9, pp. 192-200, 1998

[9] A. Stefani, W. Yuan, C. Markos, and O. Bang, "Narrow Bandwidth 850nm Fiber Bragg Gratings in Few-Mode Polymer Optical Fibers, " IEEE Photn. Tech. Lett., vol. 23, no. 10, pp. 660-662, 2011

[10] H. Y. Liu, H. B. Liu, and G.-D. Peng, "Tensile strain characterization of polymer optical fiber Bragg gratings," Opt. Commun. Vol. 251, pp. 3743, 2005.

[11] W. Zhang, D. J. Webb, G.-D. Peng, "Investigation into Time Response of Polymer Fiber Bragg Grating Based Humidity Sensors," IEEE J. Lightwave Tech. vol. 30, no. 8, pp. 1090 - 1096, 2012.

[12] W. Zhang. D. J. Webb, "Factors influencing temperature sensitivity of PMMA based optical fiber Bragg gratings," Proc. SPIE vol. 9128, p. 912821,2014

[13] W. Zhang, A. Abang, D. J. Webb, and G. -D. Peng, "An investigation into the wavelength stability of polymer optical fibre Bragg gratings," Proc. SPIE vol. 8426, p. 842619, 2012.

[14] W. Yuan, L. Khan, D. J. Webb, K. Kalli, H. K. Rasmussen, A. Stefani, O. Bang, "Humidity insensitive TOPAS polymer fiber Bragg grating sensor," Opt Express vol. 19, no. 20, pp. 19731-19739, 2011.

[15] K. T. O'Mahoneya, A.S. Mainb, D.J. Webbb, A. Martinezb and D.A. Flavin, "Implications of high power losses in IR femtosecond laser inscribed fibre Bragg gratings," Proc. SPIE vol. 6193, p. 61930Z, 2006. 